\title{
Gestão do trabalho de equipes da saúde da família
}

\author{
Family health team work management \\ Gestión del trabajo en equipo de salud familiar
}

\begin{abstract}
Josué Souza Gleriano ${ }^{1}$ [D
Gisele Caroline Richi Fabro² (1)

Wanderson Borges Tomaz ${ }^{3}$ (B)

Aldaísa Cassanho Forster ${ }^{4}$ (C)

Lucieli Dias Pedreschi Chaves ${ }^{5}$ [C]
\end{abstract}

1. Universidade do Estado de Mato Grosso, Departamento de Enfermagem. Tangará da Serra, MT, Brasil.

2. Universidade de São Paulo, Escola de Enfermagem de Ribeirão Preto, Programa de Pós-Graduação em Enfermagem Fundamental. Ribeirão Preto, SP, Brasil.

3. Universidade Federal do Triângulo Mineiro, Hospital das Clínicas da Empresa Brasileira de Serviços Hospitalares. Uberaba, MG, Brasil.

4. Universidade de São Paulo, Faculdade de Medicina de Ribeirão Preto, Departamento de Medicina Social. Ribeirão Preto, SP, Brasil.

5. Universidade de São Paulo, Escola de Enfermagem de Ribeirão Preto, Departamento de Enfermagem Geral e Especializada, Programa de Pós-Graduação em Enfermagem Fundamental. Ribeirão Preto, SP, Brasil.
Autor correspondente:

Josué Souza Gleriano

E-mail: josuegleriano@unemat.br

Recebido em 27/03/2020

Aprovado em 29/06/2020.

DOl:https://doi.org/10.1590/2177-9465-EAN-2020-0093

\section{RESUMO:}

Objetivos: Descrever o perfil, formação complementar, aspectos sobre educação permanente, vínculo e plano de carreira de profissionais de equipes da Saúde da Família. Método: Pesquisa descritiva de abordagem quantitativa. Participaram 78 membros de equipes multiprofissionais. Os dados foram coletados em 2016, por meio de um instrumento para caracterizar o profissional e as dimensões de tempo de atuação e qualificação dos profissionais das equipes, formas de contratação e modalidades de vínculos profissionais, plano de carreira e remuneração variável e educação permanente. As análises foram geradas no software IBM SPSS versão 21. Resultados: A distribuição de médicos e enfermeiros é homogênea, percebe-se uma redução de agentes comunitários de saúde. Foram mais frequentes as características: vínculo trabalhista regulado pela Consolidação das Leis Trabalhistas, baixa formação complementar para a atenção primária à saúde, até um ano de trabalho na equipe, com baixa formação complementar para área e iniciativas de ações de educação pela gestão. Conclusões e implicações para a prática: Existem fragilidades que podem interferir na organização do processo de trabalho influenciada pelos eixos da formação e formas de contratação.

Palavras-chave: Recursos Humanos; Gestão em saúde; Atenção Primária à Saúde; Saúde da Família; Enfermagem.

\section{RESUMEN}

Objetivos: Describir el perfil, la capacitación complementaria, los aspectos sobre la educación permanente, el vínculo y el plan de carrera de los profesionales de los equipos de salud familiar. Método: Investigación descriptiva con enfoque cuantitativo. Participaron 78 miembros de equipos multidisciplinarios. Los datos se recopilaron en 2016, utilizando un instrumento para caracterizar al profesional y las dimensiones de tiempo de actuación y calificación de los profesionales del equipo, formas de contratación y modalidades de vinculo profesional, plan de carrera y remuneración variable y la educación permanente. Los análisis se generaron en el software IBM SPSS versión 21. Resultados: La distribución de médicos y enfermeras es homogénea, se observa una reducción en los agentes comunitarios de salud. Las características más frecuentes fueron: vinculo de trabajo través de la Consolidación de las Leyes del Trabajo, baja capacitación complementaria para la atención primaria en salud, hasta un año de trabajo en el equipo, con baja capacitación complementaria para el área e iniciativas de acciones educativas por parte de la gerencia. Conclusiones e implicaciones para la práctica: Existen debilidades que pueden interferir en la organización del proceso de trabajo influenciado por los ejes de la capacitación y las formas de contratación.

Palabras clave: Recursos Humanos; Gestión en Salud; Atención Primaria de Salud; Salud de la Familia; Enfermería.

\section{ABSTRACT}

Objectives: To describe the profile, complementary training, aspects about permanent education, bond and career plan of professionals from Family Health teams. Method: Descriptive research with a quantitative approach. Seventy-eight members of multi-professional teams participated. Data were collected in 2016, using a tool to characterize the professional and the dimensions of time of work and qualification of the team's professionals, ways of hiring and modalities of professional bonds, career plan and variable remuneration and permanent education. The analyzes were generated by using IBM SPSS software version 21. Results: The distribution of physicians and nurses is homogeneous, one notices a reduction of community health agents. The most frequent characteristics were: legal and formal employment relationship, low complementary training for primary health care, up to one year of work in the team, with low complementary training for the area and initiatives of educational actions by management. Conclusions and implications for practice: There are weaknesses that can interfere in the organization of the work process influenced by the training axes and forms of hiring.

Keywords: Workforce; Health Management; Primary Health Care; Family Health; Nursing 


\section{INTRODUÇÃO}

No Brasil, o avanço da Atenção Primária à Saúde (APS) é fruto de intensos arranjos no aparato estatal e de forte influência internacional nos investimentos. Em 2006, o Ministério da Saúde (MS) publicou a primeira Política Nacional de Atenção Básica (PNAB), sendo a Estratégia Saúde da Família (ESF) o modelo preferencial de reorganização da APS no Sistema Único de Saúde (SUS). ${ }^{1}$

A ESF compreende a organização de processos e gestão democrática mediante a comunicação-negociação com o protagonismo dos profissionais de saúde. Assim, para a organização do trabalho em saúde na APS existe uma articulação entre recursos físicos e tecnológicos e capital humano. ${ }^{2}$ No tocante à organização de pessoal, desde a institucionalização da ESF, a diretriz previa a formação de equipes multiprofissionais com responsabilidade em abrangência territorial.

Percebe-se que, nas revisões publicadas da PNAB, houve mudanças significativas na forma de organização. ${ }^{3} A$ revisão da PNAB, publicada em 2017, momento em que se discutia a reforma do Estado, apresentou à gestão em saúde novas possibilidades de vínculos profissionais e, em particular, das formas de contratação de pessoal, que desencadeou na APS uma proliferação de vínculos empregatícios transitórios. ${ }^{4}$ Entretanto, mesmo com as mudanças na proposta inicial, a PNAB ainda é a principal diretriz, no SUS, de organização do acesso da população à APS e aos serviços de saúde. ${ }^{5}$

Destaca-se que as modificações que ocorreram na política da APS do Brasil têm sinalizado desafios para a condução da atenção à saúde. ${ }^{3}$ Nesse contexto, nos últimos anos, o trabalho na ESF tem se fragilizado na prática por ações individuais dos profissionais, além do desapreço profissional e de assalariamento, ${ }^{6}$ situação que estava sendo verificada antes das mudanças da PNAB 2017 e que, a partir de sua publicação, reforça a fragmentação por trabalho especializado na contramão do trabalho em equipe multiprofissional.

Assim, tem-se constatado na estrutura jurídico-administrativa do SUS, inclusive na APS, acelerada contratação de profissionais com salários diferentes para a realização de trabalhos semelhantes, estabelecendo multiplicidade de remunerações, vínculos e formas de contratação. ${ }^{7}$

Para a APS, as cedências na forma de vínculos e da normalização das relações de trabalho repercutem na fragilidade dos direitos trabalhistas com implicações que refletem, diretamente, na proposta do modelo de atenção. Nesse sentido, a possibilidade de estudo, a partir dos dados do Programa Nacional de Melhoria do Acesso e da Qualidade da Atenção Básica (PMAQ-AB), permite observar a situação do trabalho e do trabalhador de saúde na APS. ${ }^{8}$

Considera-se importante para o planejamento da força de trabalho a percepção do trabalhador sob o seu trabalho, que é essencial para garantir a condução do sistema de saúde, ${ }^{9}$ pois esse é coparticipante nas ações e decisões e pode favorecer estratégias específicas para a gestão em saúde local.

Desta maneira, reconhecer os processos da dinâmica da gestão do trabalho que perpassa pela educação ao trabalhador do SUS propõe à gestão em saúde ampliar estratégias que possam ser transformadoras das práticas de saúde.

A relevância da APS no âmbito da assistência e da gestão de sistemas de saúde acrescida da importância da gestão de equipes para a viabilização do trabalho na ESF e da possibilidade de contribuir para a construção de conhecimentos que subsidiem a elaboração de estratégias de qualificação do trabalho da APS e favoreçam a gestão na tomada de decisão, a fim de gerar benefícios, justificaram a realização desse estudo. Essa investigação teve como objetivo descrever o perfil, formação complementar, aspectos sobre educação permanente, vínculo e plano de carreira de profissionais de equipes da Saúde da Família.

\section{MÉTODO}

Trata-se de um estudo descritivo de abordagem quantitativa, realizado em um município do médio norte mato-grossense.

No referido município houve investimento progressivo na cobertura da APS por meio da adesão ao Programa Mais Médicos (PMM), que impulsionou o incremento quantitativo de profissionais e repercutiu em ampliação do acesso. Dados da Secretaria de Atenção Primária à Saúde do MS demostram que, em 2013, o município possuía cobertura de $47,61 \%$ de APS e que, após a adesão ao PMM, elevou a cobertura para 71,9\% em 2014, 82,2\% em 2015 e 98,3\% em 2016, configurando nesse ano o ápice de oferta de serviços na APS para a realidade local, em apenas três anos.

O presente estudo integra um conjunto de pesquisas desenvolvidas no quadriênio 2015-2018, com integração ensino-serviço e em parceria estabelecida com a gestão em saúde municipal por meio do Escritório de Qualidade para Organizações de Saúde (EsQualOS/UNEMAT). Como parte do protocolo de pesquisas ficou acordada a divulgação científica após a entrega dos relatórios finais à gestão.

Para participação no estudo, por conveniência, foram convidadas dez unidades de saúde da família cadastradas para receber a avaliação do $3^{\circ}$ ciclo do PMAQ-AB. Tal seleção de conveniência justifica-se por estarem cadastradas para avaliação do $3^{\circ}$ ciclo do PMAQ e possuírem equipe multidisciplinar completa no período da coleta de dados da pesquisa. Quanto aos membros da equipe, todos foram convidados e aceitaram participar do estudo.

Setenta e oito membros da equipe multiprofissional (enfermeiro, médico, técnicos de enfermagem, recepcionista, agentes comunitários de saúde (ACS), cirurgião-dentista e técnico de higiene bucal) participaram do estudo. Os dados foram coletados no segundo semestre de 2016, em visita presencial dos pesquisadores responsáveis em cada unidade de saúde, após agendamento por telefone, de acordo com o melhor dia e horário para a equipe participar. Guiou-se a coleta por meio de um instrumento estruturado de perfil sociodemográfico e um disponível no guia do $P M A Q^{10}$ Módulo II - Entrevista com Profissional da Equipe de Atenção Básica e Verificação de Documentos na Unidade Básica de Saúde. Nesse estudo, foram utilizados os tópicos: tempo de atuação, formação complementar e qualificação dos profissionais das equipes da saúde da família, formas de contratação e modalidades de vínculos profissionais, plano de carreira e remuneração variável e educação permanente, que estão sistematizadas no Quadro 1.

Após a coleta dos dados, houve dupla digitação em planilha eletrônica, na qual as análises foram geradas por meio do software IBM SPSS ${ }^{\circledR}$ versão 21 e apresentadas em tabelas com seus valores absolutos e percentuais. Para fins de análise 
e discussão, os resultados foram apresentados em blocos, conforme as dimensões selecionadas no Quadro 1.

Foram respeitados todos os aspectos éticos em pesquisa, de acordo com a Resolução 466/12, com apreciação e aprovação do Comitê de Ética em Pesquisa com Seres Humanos (CEP) sob o no CAEE: 51340215.0.00005166.

\section{RESULTADOS}

Os resultados são apresentados em quatro tabelas: a Tabela 1 exibe o quantitativo estratificado do perfil por profissão, coordenação de equipe, tempo de atuação dos membros na equipe de saúde, formação complementar e o tipo de formação; a Tabela 2 apresenta

Quadro 1. Variáveis segundo dimensões de análise da gestão do trabalho na Atenção Básica.

\begin{tabular}{|c|c|}
\hline DIMENSÕES DE ANÁLISE & VARIÁVEIS \\
\hline $\begin{array}{l}\text { Tempo de atuação, } \\
\text { perfil e formação dos } \\
\text { profissionais da equipe } \\
\text { de APS }\end{array}$ & $\begin{array}{l}\text { Profissão do entrevistado } \\
\text { O senhor é coordenador da equipe? } \\
\text { Quanto tempo o senhor atua nesta equipe de AB? } \\
\text { O senhor possui ou está em formação complementar? } \\
\text { Possui quais destes processos de formação? }\end{array}$ \\
\hline Educação Permanente & $\begin{array}{l}\text { Há no município ações de educação permanente que envolvam profissionais da Atenção Básica? } \\
\text { Essas ações de educação permanente contemplam as demandas e necessidades da equipe? } \\
\text { Em quais dessas ações a equipe participa ou participou no último ano? }\end{array}$ \\
\hline $\begin{array}{l}\text { Formas de contratação e } \\
\text { modalidade de vínculos } \\
\text { profissionais }\end{array}$ & $\begin{array}{l}\text { Qual o seu agente contratante? } \\
\text { Qual é seu tipo de vínculo? }\end{array}$ \\
\hline $\begin{array}{l}\text { Plano de carreira e } \\
\text { Remuneração variável }\end{array}$ & $\begin{array}{l}\text { O senhor tem plano de carreira? } \\
\text { No plano tem progressão por tempo? } \\
\text { No plano tem progressão segundo avaliação de desempenho ou desenvolvimento (mérito)? } \\
\text { Gratificação por formação/titulo? } \\
\text { O senhor tem incentivo, gratificação, prêmio financeiro por desempenho? }\end{array}$ \\
\hline
\end{tabular}

Fonte: Adaptado do documento - Saúde mais perto de você: acesso e qualidade Programa Nacional de Melhoria do Acesso e da Qualidade da Atenção Básica (PMAQ) Documento síntese para avaliação externa, publicado pelo Ministério da Saúde ${ }^{10}$

Tabela 1. Distribuição dos membros das equipes de APS, segundo o perfil profissional, as categorias de participação e tipo de formação complementar, de um município do médio norte mato-grossense, 2016

\begin{tabular}{|c|c|c|}
\hline & $\mathbf{N}$ & $\%$ \\
\hline \multicolumn{3}{|l|}{ Profissão } \\
\hline Médico & 10 & 12.82 \\
\hline Enfermeiro & 10 & 12.82 \\
\hline Cirurgião-dentista & 07 & 8.97 \\
\hline Técnico de Enfermagem & 10 & 12.82 \\
\hline Técnico de Higiene Bucal & 07 & 8.97 \\
\hline Recepcionista & 10 & 12.82 \\
\hline Agente Comunitário de Saúde & 24 & 30.78 \\
\hline \multicolumn{3}{|l|}{ Coordenação da Equipe } \\
\hline Enfermeiro & 10 & 100 \\
\hline \multicolumn{3}{|l|}{ *Tempo de atuação dos profissionais na equipe } \\
\hline 1 ano & 52 & 66,66 \\
\hline 2 anos & 07 & 12.82 \\
\hline 3 anos ou mais & 19 & 20.76 \\
\hline \multicolumn{3}{|l|}{ *Formação complementar } \\
\hline Profissionais de nível superior que possuem ou estão em formação complementar & 15 & 55,55 \\
\hline \multicolumn{3}{|l|}{ *Tipo de Formação complementar } \\
\hline Especialização em Medicina de Família e Comunidade/Saúde da Família & 04 & 26.66 \\
\hline Outras especializações & 09 & 73.34 \\
\hline
\end{tabular}

Fonte: Extraído do banco de dados da pesquisa. * Adaptado do documento:Saúde mais perto de você: acesso e qualidade Programa Nacional de Melhoria do Acesso e da Qualidade da Atenção Básica (PMAQ) Documento síntese para avaliação externa ${ }^{10}$ 
os resultados referentes à Educação Permanente (EP); a Tabela 3 se refere às formas de contratação e modalidade de vínculo trabalhista dos profissionais; e a Tabela 4 apresenta o panorama dos planos de carreiras nos municípios.

Percebe-se que a coordenação das unidades é feita por enfermeiros, com maior frequência, que as equipes possuem pouco tempo de atuação na área de abrangência e que a formação complementar na área da APS é baixa. Embora existissem momentos de ações de capacitação, os participantes apontaram que os temas abordados não contemplaram as necessidades do trabalho. Observa-se a ausência de aspectos relativos ao plano de carreira, considerando incentivos, gratificação, prêmio financeiro por avaliação de desempenho e/ou progressão por titulação e formação profissional.

Tabela 2. Distribuição das respostas dos membros das equipes de APS em relação à Educação Permanente (EP) de um município da região médio norte mato-grossense, 2016

\begin{tabular}{lcc}
\hline & N & $\%$ \\
\hline Existência de ações de educação permanente & 63 & 80,76 \\
\hline Ações que contemplam as necessidades de capacitação & 42 & 66,66 \\
*Tipo de ações de educação permanente no último ano** & & \\
\hline Telessaúde & 26 & 19,40 \\
\hline Telediagnóstico & 8 & 5,97 \\
\hline Teleconsultoria & 12 & 8,95 \\
\hline EAD/UNASUS & 23 & 17.16 \\
\hline Cursos presenciais & 63 & 47.01 \\
\hline Tutoria/preceptoria & 2 & 1,49 \\
\hline
\end{tabular}

Fonte: Extraído do banco de dados da pesquisa. * Adaptado do documento: Saúde mais perto de você: acesso e qualidade Programa Nacional de Melhoria do Acesso e da Qualidade da Atenção Básica (PMAQ) Documento síntese para avaliação externa ${ }^{10},{ }^{* *}$ Na variável, admitiram-se múltiplas respostas

Tabela 3. Distribuição dos membros das equipes de APS segundo o tipo de vínculo de um município da região médio norte mato-grossense, 2016

\begin{tabular}{lcc}
\hline & $\%$ & $\mathrm{~N}$ \\
\hline *Trabalhadores que relatam que têm como agente contratante & 78 & 100 \\
\hline Administração direta & - \\
\hline Terceirização & 4 \\
*Tipo de vínculo & 5.12 \\
\hline Servidores públicos estatutários & 3.84 \\
Contrato CLT & 3 \\
Contrato temporário pela administração pública regido por legislação especial (federal, & 71 \\
estadual, municipal) & 91.04 \\
\hline
\end{tabular}

Fonte: Extraído do banco de dados da pesquisa. * Adaptado do documento: Saúde mais perto de você: acesso e qualidade Programa Nacional de Melhoria do Acesso e da Qualidade da Atenção Básica (PMAQ) Documento-síntese para avaliação externa ${ }^{10}$

Tabela 4. Distribuição do panorama dos planos de carreiras dos membros das equipes de APS de um município da região médio norte mato-grossense, 2016

\begin{tabular}{lcc}
\hline & N & $\%$ \\
\hline *Profissionais possuem plano de carreira & 4 & 5.12 \\
\hline *Profissionais têm incentivo, gratificação, prêmio financeiro por desempenho & - & - \\
*Profissionais possuem no plano de carreira progressão por antiguidade & 4 & 5.12 \\
*Progressão segundo avaliação de desempenho ou desenvolvimento & - & - \\
\hline *Progressão por titulação e formação profissional & - & - \\
\hline
\end{tabular}

Fonte: Extraído do banco de dados da pesquisa. * Adaptado do documento: Saúde mais perto de você: acesso e qualidade Programa Nacional de Melhoria do Acesso e da Qualidade da Atenção Básica (PMAQ) Documento síntese para avaliação externa ${ }^{10}$ 


\section{DISCUSSÃO}

Participaram das entrevistas 68 profissionais de saúde e 10 recepcionistas. Destes, 12,8\% eram enfermeiros, $12,8 \%$ médicos, $8.9 \%$ cirurgiões-dentistas, $12,8 \%$ técnicos de enfermagem, $8.9 \%$ técnicos de higiene bucal, 30,7\% ACS e $12,8 \%$ recepcionistas.

Todos os profissionais enfermeiros assumem a coordenação das equipes de APS, o que gera duplicidade de atividades, gestão e assistência. Na América Latina, emergem discussões para o avanço na normatização da atuação em enfermagem, propondo práticas avançadas e de formação mínima, ${ }^{11} \mathrm{com}$ vistas a caracterizar possível aumento do escopo de prática dos enfermeiros, o que pode contribuir para a melhoria do acesso e da cobertura em saúde. ${ }^{12}$

Com relação ao tempo de atuação desses membros, observa-se que $66,6 \%$ dos entrevistados informaram ter menos de um ano de atuação na equipe e $12.8 \%$, até dois anos.

As contratações realizadas nesse contexto não asseguram fixação. No Brasil, estudos sobre recursos humanos na APS demostram alta rotatividade, ${ }^{5}$ pouco incentivo para a progressão salarial e profissional alinhado às baixas condições de trabalho e envolvimento dos profissionais na transformação do modelo de atenção, justificado por formação incipiente, ${ }^{13,14}$ que compromete a efetividade do modelo assistencial pensado para a APS, ${ }^{15}$ além de prejudicar a qualidade da assistência e a satisfação dos usuários. ${ }^{16}$

Os ACS, que representam força de trabalho essencial à ESF, tiveram, na composição da equipe de saúde, forte alteração, segundo a PNAB de 2017. ${ }^{17}$ Ademais, questiona-se a qualificação desse profissional em assumir as atribuições de orientação e acompanhamento das múltiplas condições de saúde. ${ }^{18}$ Percebe-se que foram oportunizadas diversas formas de qualificação breves e intermitentes ao ACS e, embora tenha configurado alternativa à demanda dos serviços, representou, em certo momento, um espaço de avanço que não se confirmou. ${ }^{19}$

Outro aspecto a ser considerado nesse município diz respeito ao investimento progressivo da cobertura da APS, que caracterizou ampliação do acesso, a partir do ano de 2014, após provisão emergencial de médicos pelo PMM, o que requereu da gestão local a organização dos serviços e incremento quantitativo e fixação de médicos e, consequentemente, de outros profissionais para o nível primário. Entretanto, houve decréscimo de cobertura nos anos subsequentes: 2017 foi de $85,4 \%$, 83,9\% em 2018 , e até novembro de 2019, a cobertura registrada era de $80,2 \%$, em 22 unidades de Saúde da Família. ${ }^{20}$

Sobre a formação complementar, $55 \%$ dos profissionais de nível superior declararam ter concluído ou estar cursando alguma pós-graduação, somente $10 \%$ dos médicos e $30 \%$ dos enfermeiros declararam ter concluído especializações na área da APS.

Destaca-se que $90 \%$ dos médicos declararam ter concluído residência em Clínica Médica ou Cirúrgica e nenhum entrevistado referiu quaisquer residências em Saúde da Família ou Medicina de Família e Comunidade e Residências em Saúde Pública/Saúde Coletiva.

Para a qualificação do processo de trabalho em saúde, valorização e satisfação do trabalhador, o Ministério da Saúde tem incentivado a gestão a implementar atividades de Educação Permanente em Saúde (EPS). Assim, a EPS pode ser entendida como um dispositivo potente de gestão do trabalho e está ancorada na Política Nacional da Educação Permanente em Saúde (PNPS). ${ }^{21}$

Dentre os entrevistados, 80,7\% afirmaram participar de momentos de ações de capacitação ofertadas pela gestão municipal, porém, o maior quantitativo dos profissionais sinalizou que os temas abordados não contemplaram as necessidades de capacitação. Nesse aspecto, é necessário rever como têm sido capilarizadas as demandas que os profissionais têm feito em âmbito operacional, ou como a análise de encaminhamentos às teleconsultorias é realizada. Dentre os que afirmaram terem participado das ações de educação permanente, as modalidades mais frequentes foram os cursos presenciais, o que reforça a necessidade de expandir o uso da plataforma de educação da telessaúde nesses serviços.

Na perspectiva de um município no interior do estado de Mato Grosso, com uma rede que possui nos serviços estrutura informatizada com acesso à internet, o incentivo no uso da telessaúde pode possibilitar, no âmbito da gestão, direcionamento para o plano de educação permanente e, na perspectiva dos profissionais, capacidade de institucionalizar, no serviço, estratégias de ensino, as quais podem ser compartilhadas na equipe.

Nesse sentido, para a superação da extensão territorial e das iniquidades de distribuição de serviços, a telessaúde possibilita utilizar recursos tecnológicos para a interação entre os profissionais de saúde e a rede de serviços, podendo promover, para além de aprimoramento do trabalho da equipe, acesso a recursos de apoio diagnóstico e terapêutico, ampliando a coordenação do cuidado da APS. ${ }^{22}$

Profissionais reconheceram que o uso dos serviços de Tele-educação foi atribuído à sua aplicação no apoio assistencial. ${ }^{23}$ A contribuição da telessaúde, como dispositivo de apoio às equipes de saúde, potencializa a ampliação do acesso e da qualidade da atenção. ${ }^{24,25}$

Nesse sentido, a oferta da educação permanente para as equipes pode contribuir para o fortalecimento e ampliação do acesso à APS, por meio da qualificação para os profissionais.

Quanto à contratação, o maior percentual é de contratos temporários diretos com a administração pública, o que sugere maior fragilidade de vínculos trabalhistas. Tal fato pode representar limites para a construção do processo de trabalho em unidades de saúde, como modelo de saúde da família, o que pressupõe estabelecimento de vínculos.

Em municípios de maior porte populacional na APS, a terceirização, flexibilização dos vínculos trabalhistas e contratação por CLT são mais presentes. ${ }^{26}$ Contudo, processos de flexibilização mudam, significativamente, o contexto do trabalho no campo da saúde. ${ }^{27}$ 
Especificamente, para o ACS há intensificação da realização de atividades administrativas em detrimento do tempo para visitas domiciliares somado a uma proposta de trabalho para a coleta de informações e preenchimento de sistemas de informação, perfazendo um tensionamento em desiguais vínculos empregatícios e precárias condições de trabalho com sub-remuneração. ${ }^{28}$

Dados os limites impostos pela política econômica, importa lembrar que há mecanismos de contratação de pessoal por meio de Organizações Não-Governamentais (ONGs) e Organizações da Sociedade Civil de Interesse Público (Oscip), cujos dispêndios são contabilizados como "serviços de terceiros" e não como "despesas de pessoal, ${ }^{29}$ dispositivo utilizado para burlar a Lei de Responsabilidade Fiscal". O que depreende a necessidade de um mecanismo para criar possibilidades de ampliar os gastos, sem confrontar-se com a lei de responsabilidade fiscal e respeitar a contratação.

Não foram identificados aspectos relativos ao plano de carreira, considerando incentivos, gratificação, prêmio financeiro por avaliação de desempenho e/ou progressão por titulação e formação profissional. Entretanto, destaca-se que quatro servidores estatutários se enquadravam em critério de progressão na carreira por tempo de serviço. Apenas por plano de carreira, quatro servidores, por serem estatutários, enquadravam-se na progressão por antiguidade.

A luta de classes trabalhadoras levou ao reconhecimento de direitos garantidos constitucionalmente, sendo o plano de carreira uma potente ferramenta de gestão, principalmente quando agregado a outros subsídios, como a gratificação, a avaliação de desempenho e a progressão por qualificação, ${ }^{7}$ porém, percebem-se limitações para a implementação no setor saúde.

Pode-se afirmar que a incorporação dos Planos de Cargos, Carreiras e Salários (PCCS) nos municípios ainda é frágil. Reconhece-se interferência estatal na construção de um plano de carreira para a ESF, além da inexistência de PCCS ou incipiência na sua instituição, principalmente em municípios de menor porte..$^{30}$ Porém, o PCCS é um instrumento que mobiliza e potencializa a motivação dos trabalhadores, contribui para a valorização, fixação e perspectiva de carreira no serviço. ${ }^{31}$

Os limites já reconhecidos de dificuldades de atração e fixação de profissionais e a não consolidação do plano de carreira, cargos e salários como instrumento estratégico para a gestão do trabalho ${ }^{29}$ têm sido trabalhados pela Secretaria de Gestão do Trabalho e da Educação na Saúde (SGTES) por meio de projetos de cooperação técnica com municípios e estados para alavancar iniciativas no fortalecimento do Programa de Desprecarização do Trabalho no SUS (DesprecarizaSUS), porém, ainda faltam demonstrações de capacidade de acompanhamento e avaliação, além de gestores reconhecidas de $\mathrm{RH}$ desconhecerem o DesprecarizaSUS. ${ }^{32}$

No entanto, essas questões são como desafios importantes para a gestão e força de trabalho na APS do SUS, capazes de impactar, consideravelmente, a organização do modelo de atenção, do processo de trabalho e da efetividade das práticas de atenção à saúde.
Considera-se que a consolidação da APS, nos últimos anos, é um dos movimentos mais relevantes no avanço da universalidade no SUS, sendo a ESF o eixo que tem ancorado esse processo. Embora o município estudado possa ter conseguido a expansão da APS por meio de investimentos federais e municipais após o PMM, as fragilidades de contratação e os ajustes fiscais na redução dos gastos em proteção social, dado o cenário nacional, abriram brechas para a flexibilização da dedicação dos membros da equipe multiprofissional, redução de carga horária e diminuição da presença do ACS na equipe.

\section{CONSIDERAÇÕES FINAIS E IMPLICAÇÕES PARA A PRÁTICA}

Nesse estudo, os profissionais possuíam pouco tempo de atividade na unidade, baixa formação na área e vínculos de trabalho frágeis, o que repercute na força de trabalho da APS nesse município. Há impactos para a condução e organização da atenção, embora tenha ampliado a cobertura.

O enfermeiro é um articulador da APS e da dupla ocupação de coordenação da equipe e assistência, porém precisa estar atento no acúmulo de funções exercidas por esses profissionais e para as múltiplas tarefas que exercem.

A formação para a APS ainda é incipiente, o que configura fragilidades para a efetividade da atenção e empenho na mudança de cultura da própria população. A qualificação é emergente, nesse caso, para promover espaços de melhoria do processo de trabalho. Assim, o uso de ações de educação permanente por parte da gestão poderá auxiliar na mudança desse perfil.

Destaca-se a precarização dos vínculos de trabalho, o que pode ocasionar rotatividade e descontinuidade de ações nas equipes. Nesse caso, é necessário retomar o diálogo no sentido da organização do processo de trabalho e de estratégias que auxiliem a dar continuidade ao cuidado, frente a essas situações expostas.

Na gestão do trabalho em saúde a valorização do trabalhador da saúde e do seu trabalho, por meio dos PCCS, é uma das estratégias que podem fixar e engajar a efetividade no desempenho do trabalho em saúde, porém, há um crescente avanço de iniciativas neoliberais, que reverberam em possibilidades mais efetivas de investimento no capital humano.

Como contribuição, o estudo mostra os nuances da realidade da gestão local para articular a política de recursos humanos na implementação da APS. Reforça eixos estratégicos na gestão em saúde, como a educação permanente, a telessaúde e os desafios de interiorizar serviços frente a aspectos políticos institucionais que podem, ou não, favorecer a fixação de profissionais.

As limitações desse estudo estão relacionadas com a compreensão do cenário local e, embora tais limitações imprimam a realidade exposta para pensar em diretrizes para lidar com esses recursos humanos, corroboram os achados que ganham concretude em diversas regiões do país, cujos cenários são peculiares como esse, e podem auxiliar o estado e o Ministério da Saúde (MS) por meio da Secretaria de Gestão do Trabalho 
e Educação na Saúde (SGTES) a adotar estratégias indutoras de qualificação.

Neste sentido, recomenda-se a realização de novos estudos em outros cenários para conhecer e explorar o uso dos dados captados pelas avaliações do PMAQ -AB.

\section{CONTRIBUIÇÃO DOS AUTORES}

Desenho do estudo. Aquisição, análise de dados e interpretação dos resultados. Revisão de literatura. Redação e revisão crítica do manuscrito. Aprovação da versão final do artigo. Responsabilidade por todos os aspectos do conteúdo e integridade do artigo publicado. Josué Souza Gleriano

Análise de dados e interpretação dos resultados. Revisão de literatura. Redação e revisão crítica do manuscrito. Aprovação da versão final do artigo. Responsabilidade por todos os aspectos do conteúdo e integridade do artigo publicado. Gisele Caroline Riche Fabro

Análise de dados e interpretação dos resultados. Redação e revisão crítica do manuscrito. Aprovação da versão final do artigo. Responsabilidade por todos os aspectos do conteúdo e integridade do artigo publicado. Wanderson Borges Tomaz Aldaísa Cassanho Forster Lucieli Dias Pedreschi Chaves

\section{EDITOR ASSOCIADO}

\section{Maria Catarina Salvador da Motta}

\section{REFERÊNCIAS}

1. Portaria № 648/GM de 28 de março de 2006 (BR). Aprova a Política Nacional de Atenção Básica, estabelecendo a revisão de diretrizes e normas para a organização da Atenção Básica para o Programa Saúde da Família (PSF) e o Programa Agentes Comunitários de Saúde (PACS). Diário Oficial da União [periódico na internet], Brasília (DF), 2006 [citado 2020 jan 25]. Disponível em: http://bvsms.saude.gov.br/ bvs/publicacoes/prtGM648_20060328.pdf

2. Trindade LL, Pires DEP. Implications of primary health care models in workloads of health professionals. Texto Contexto Enferm. 2013;22(1):3642. http://dx.doi.org/10.1590/S0104-07072013000100005.

3. Morosini MVGC, Fonseca AF, Lima LD. Política Nacional de Atenção Básica 2017: retrocessos e riscos para o Sistema Único de Saúde. Rev Saúde em debate. 2018;42(116):11-24. https://doi.org/10.1590/01031104201811601

4. Donadone JC, Baggenstoss S. Intermediários e as novas configurações no tecido organizacional brasileiro: Um estudo sobre as organizações sociais de saúde. Tempo soc. 2017;29(1):130-49. https://doi. org/10.11606/0103-2070.ts.2017.2017.120270.

5. Pires DEP, Vandresen L, Forte ECN, Machado RR, Melo TAP. Management in primary health care: implications on managers workloads. Rev Gaúcha Enferm. 2019;40:e20180216. http://dx.doi.org/10.1590/19831447.2019.20180216. PMid:31596341.

6. Fontana KC, Lacerda JT, Machado PMO. O processo de trabalho na Atenção Básica à saúde: avaliação da gestão. Saúde Debate. 2016;40(110):64-80. http://dx.doi.org/10.1590/0103-1104201611005

7. Antoni CR. As reformas do Estado, da saúde e recursos humanos: limites e possibilidades. Ciênc saúde coletiva. 2001;6(2):341-60. https:// doi.org/10.1590/S1413-81232001000200006.

8. Bezerra MM, Medeiros KR. Limites do Programa de Melhoria do Acesso e da Qualidade da Atenção Básica (PMAQ-AB): em foco, a gestão do trabalho e a educação na saúde. Saúde debate. 2018;42(spe 2):188202. https://doi.org/10.1590/0103-11042018s213.
9. Rizzotto MLF, Gil CRR, Carvalho M, Fonseca ALN, Santos MF. Força de trabalho e gestão do trabalho em saúde: revelações da Avaliação Externa do Programa Nacional de Melhoria do Acesso e da Qualidade da Atenção Básica no Paraná. Saúde debate. 2014;38(spe):237-51. http://dx.doi.org/10.5935/0103-1104.2014S018.

10. Ministério da Saúde (BR), Programa Nacional de Melhoria do Acesso e da Qualidade da Atenção Básica (PMAQ). Saúde mais perto de você - Acesso e qualidade programa nacional de melhoria do acesso e da qualidade da atenção básica (PMAQ). Documento síntese para avaliação externa [Internet]. Brasília: Ministério da Saúde;2012 [citado 2020 jan 25]. Disponível em: http://189.28.128.100/dab/docs/sistemas/ pmaq/Documento_Sintese_Avaliacao_Externa_2012_04_25.pdf

11. Miranda No MV, Rewa T, Leonello VM, Oliveira MAC. Advanced practice nursing: a possibility for Primary Health Care? Rev Bras Enferm. 2018;71(Suppl 1):716-21. http://dx.doi.org/10.1590/0034-7167-20170672. PMid:29562033.

12. Maier CB, Aiken L. Expanding clinical roles for nurses to realign the global health workforce with population needs: a commentary. Isr J Health Policy Res. 2016;5(1):21. http://dx.doi.org/10.1186/s13584016-0079-2. PMid:27280014

13. Mendonça MHM, Martins MIC, Giovanella L, Escorel S. Desafios para gestão do trabalho a partir de experiências exitosas de expansão da Estratégia de Saúde da Família. Cienc Saúde Coletiva. 2010;15(5):235565. http://dx.doi.org/10.1590/S1413-81232010000500011.

14. Barbosa LG, Damasceno RF, Silveira DMML, Costa SM, Leite MTS Recursos Humanos e Estratégia Saúde da Família no norte de Minas Gerais: avanços e desafios. Cad Saude Colet. 2019;27(3):287-94 http://dx.doi.org/10.1590/1414-462x201900030084.

15. Seidl H, Vieira SP, Fausto MCR, Lima RCD, Gagno J. Gestão do trabalho na Atenção Básica em Saúde: uma análise a partir da perspectiva das equipes participantes do PMAQ-AB. Saúde em debate. 2014;38(spe):94108. https://doi.org/10.5935/0103-1104.2014S008.

16. Vieira JMR, Garnelo L, Hortale VA. Análise da atenção básica em cinco municípios da amazônia ocidental, com ênfase no Programa Saúde da Família. Saude Soc. 2010;19(4):825-65. http://dx.doi.org/10.1590/ S0104-12902010000400012.

17. Portaria ํㅜ 2.436, de 21 de setembro de 2017 (BR). Estabelece a revisão de diretrizes para a organização da Atenção Básica, no âmbito do SUS. Diário Oficial da União [periódico na internet], Brasília (DF), 2017 [citado 2020 jan 25]. Disponível em: http://bvsms.saude.gov.br/ bvs/saudelegis/gm/2017/prt2436_22_09_2017.html

18. Alonso CM, Béguin 2nd PD, Duarte FJCM. Work of community health agents in the Family Health Strategy: meta-synthesis. Rev Saude Publica. 2018;52:14. http://dx.doi.org/10.11606/S1518-8787.2018052000395. PMid:29489997.

19. Morosini MV, Fonseca AF. Community workers in Primary Health Care in Brazil: an inventory of achievements and challenges. Saúde debate. 2018;42(spe 1):261-74. https://doi.org/10.1590/0103-11042018s117.

20. Ministério da Saúde (BR), Secretaria de Atenção Primária à Saúde. Dispõe sobre Cobertura da atenção básica. Brasília: Ministério da Saúde; 2020 [citado 2020 jan 25]. Disponível em: https://egestorab.saude.gov. $\mathrm{br} /$ paginas/acessoPublico/relatorios/relHistoricoCoberturaAB.xhtml

21. Portaria $\mathrm{n}^{\circ} \mathbf{1 . 9 9 6}$, de 20 de agosto de 2007 (BR). Dispõe sobre as diretrizes para a implementação da Política Nacional de Educação Permanente em Saúde. Diário Oficial da União [periódico na internet], Brasília (DF), 2007 [citado 2020 jan 25]. Disponível em: http://bvsms. saude.gov.br/bvs/saudelegis/gm/2007/prt1996_20_08_2007.html

22. Piropo TGN, Amaral HOS. Telessaúde, contextos e implicações no cenário baiano. Saúde Debate. 2015;39(104):279-87. http://dx.doi. org/10.1590/0103-110420151040413.

23. Nilson LG, Calvo MCM, Dolny LL, Natal S, Maeyama MA, Lacerda JT. Avaliação da utilização de telessaúde para apoio assistencial na atenção primária à saúde. Braz J Hea Rev. 2019;2(6):6188-206. http:// dx.doi.org/10.34119/bjhrv2n6-114.

24. Bashshur RL, Howell JD, Krupinski EA, Harms KM, Bashshur N, Doarn CR. The Empirical Foundations of Telemedicine Interventions in Primary Care. Telemed J E Health. 2016;22(5):342-75. http://dx.doi.org/10.1089/ tmj.2016.0045. PMid:27128779. 
25. Harzheim E, Chueiri PS, Umpierre RN, Gonçalves MR, Siqueira ACS, D'Avila OP et al. Telessaúde como eixo organizacional dos sistemas universais de saúde do século XXI. Rev Bras Med Fam Comunidade. 2019;14(41):1881. http://dx.doi.org/10.5712/rbmfc14(41)1881.

26. Ministério da Saúde (BR), Secretaria de Gestão do Trabalho e da Educação na Saúde, Departamento de Gestão e da Regulação do Trabalho em Saúde. Precarizacão e qualidade do emprego no Programa Saúde da Família [Internet]. Belo Horizonte: Universidade Federal de Minas Gerais; 2007 [citado 2020 jan 25]. Disponível em: https://www. nescon.medicina.ufmg.br/biblioteca/imagem/2464.pdf

27. Garrido-Pinzón J, Bernardo MH. Vivências de trabalhadores da saúde em face da lógica neoliberal: um estudo da atenção básica na Colômbia e no Brasil. Cad Saude Publica. 2017;33(9):e00050716. http://dx.doi. org/10.1590/0102-311x00050716. PMid:28977271.

28. Nogueira ML. Expressões da precarização no trabalho do agente comunitário de saúde: burocratização e estranhamento do trabalho. Saude Soc. 2019;28(3):309-23. http://dx.doi.org/10.1590/s0104-12902019180783.
29. Fagnani E. Equity and health regionalization in the countercurrent. Cad Saude Publica. 2019;35(35, Suppl 2):e00120819. http://dx.doi. org/10.1590/0102-311x00120819. PMid:31644688.

30. Giovanella L, Almeida PF. Atenção primária integral e sistemas segmentados de saúde na América do Sul. Cad Saude Publica. 2017;33(33, Suppl 2):e00118816. http://dx.doi.org/10.1590/0102311x00118816. PMid:28977122.

31. Mendes TMC, Oliveira RFS, Mendonça JMN, Medeiros A Jr, Castro JL Position Plans, Careers and Salaries: perspective of health professionals from the Central-West of Brazil. Saúde Debate. 2018;42(119):849-61. http://dx.doi.org/10.1590/0103-1104201811905.

32. Junqueirai TS, Cotta RMM, Gomes RC, Silveira SFR, Siqueira-Batista R, Pinheiro TMM et al. Saúde, democracia e organização do trabalho no contexto do Programa Saúde da Família: desafios estratégicos. Rev Bras Educ Med. 2009;33(1):122-33. http://dx.doi.org/10.1590/ S0100-55022009000100016. 